\title{
Long-Term Priming of Visual Search Prevails Against Time and Instructions Supplemental Material
}

\author{
Wouter Kruijne \& Martijn Meeter \\ Vrije Universiteit Amsterdam
}

\section{Graphs of normalized measures}

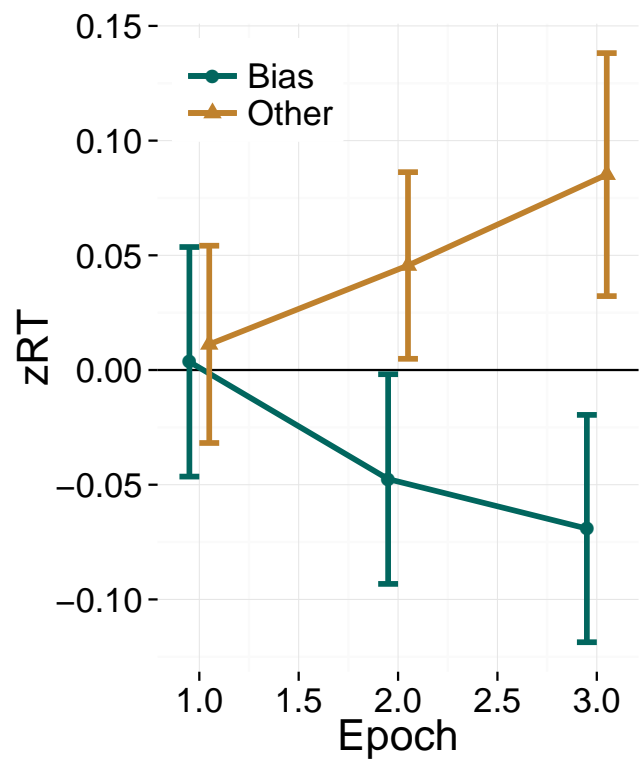

Figure 1. The color-corrected zRTs for the bias- and other color in the critical Block 4 of Experiment 1. These data were used in the the primary analysis of Experiment 1. 


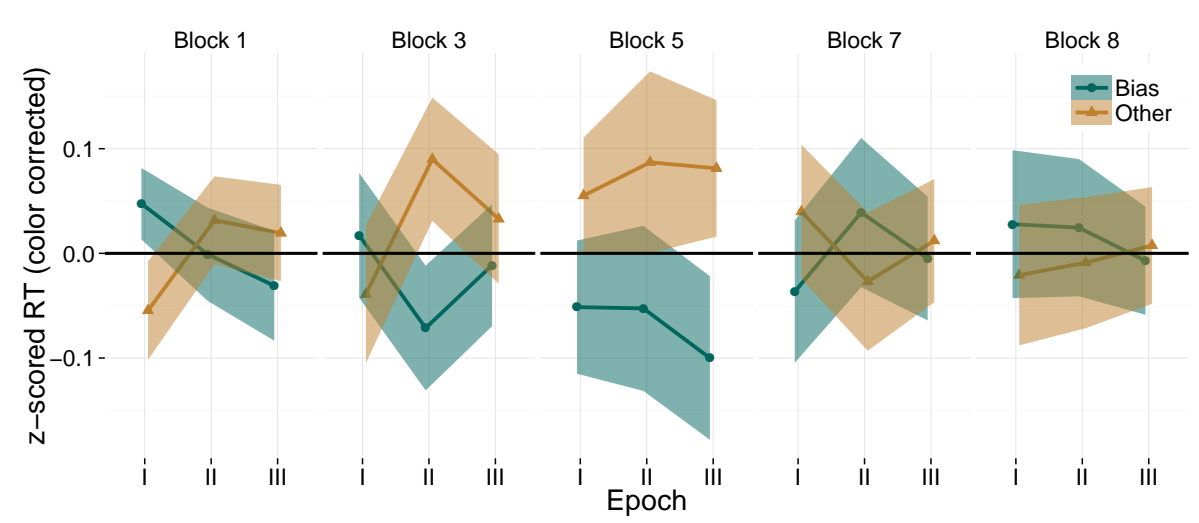

Figure 2. Color-corrected zRTs in all neutral blocks of Experiment 2. Note that Block 1 is not preceded by a color bias, and that Blocks 5-8 occur in the second session a week later. Blocks 7 and 8 were completed right after an inversed bias block. 